\title{
Social Work Practice issues with Intravenous Drug Users in India
}

\author{
${ }^{1}$ Varsha, ${ }^{2}$ Akash Gulalia

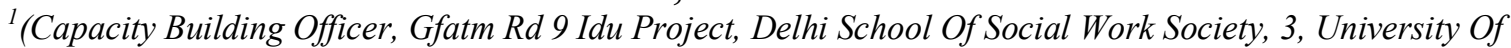 \\ Delhi, India) \\ ${ }_{2}^{2}$ (Professor And Director, Gfatm Rd 9 Idu Project, Delhi School Of Social Work Society, 3, University Of Delhi, \\ India)
}

\begin{abstract}
Drug abuse is a global phenomenon. The use of dependence producing substances, in some form or the other, is universal. In India, the use of opium and cannabis has been in existence since long ago. In the last three decades, however new drugs like heroin, amphetamine type stimulants (ATS), cocaine and pharmaceutical compounds have made their entry and are being used especially in metropolitan cities. The Constitution of India under the Article 47 of Directive Principles of State Policy directs the State to endeavour to bring about prohibition of consumption, except for medicinal purposes, of intoxicating drinks and drugs which are injurious to health. The Government of India is a signatory to three UN Conventions on the subject and several laws to control illicit use of drugs have been enacted. Recently pharmaceutical drugs injecting has increased in several parts of the country. Injecting drugs behaviour among drug addicts has increased the prevalence of HIV and other associated harms. Government is fighting back with demand and supply reduction policies and programmes by Ministry of social justice and empowerment and harm reduction and biomedical and behavioural interventions through Non government Organisations. At all levels from policy making to outreach activities professional social worker are employed by government and private organisations. This article discusses various issues related to pharmaceutical drug abuse and its implications for HIV prevention programmes in India.
\end{abstract}

Key words: Pharmaceutical drugs abuse, Intravenous Drug Use, Social Work, HIV Prevention

\section{Introduction}

Drug abuse is a global phenomenon. The use of dependence producing substances in some form is universal. The use of opium and cannabis has been in existence since long ago in India. In the last three decades, however new drugs like heroin, amphetamine type stimulants (ATS), cocaine and pharmaceutical compounds have made their entry and are being used especially in metropolitan cities. Currently, India is not merely a country for the transit of such drugs from the 'Golden Triangle' or 'Golden Crescent'; it has also become a country of consumption. The geographic location of India, wider availability and usage of drugs in the country, a socially varied mix of users, cutting across caste, class and creed boundaries, and the breakdown of traditional values resulting in part from large scale rural-urban migration, have contributed to the rise in the number of drug abusers in recent years.[1]

Drug abuse has a negative impact on every aspect of user's life - physical health, social and family life, occupation, finances etc. Therefore, drug abuse has to be viewed as a bio-psycho-social problem, which requires a combination of medical treatment and psycho-social intervention in India. Regular consumption of intoxicating psychoactive substances leads to dependence, and has serious public health consequences. It compromises both individual and social development, ruins the lives of individuals, devastates families, and damages the fabric of communities. It contributes to the burden of disease and is a leading risk factor for premature deaths and disabilities in India. Some of these compounds may lead to neuro-psychiatric disorders and other non-communicable diseases such as cardiovascular diseases, accidents, suicides and violence.[2,3]

\section{Extent pattern and trend of drug abuse}

As per the National Survey of Extent, Pattern and Trend of Drug Abuse in India, sponsored by the Ministry of Social Justice and Empowerment and by the United Nations Office on Drugs and Crime, Regional Office South Asia (UNODC-ROSA) in 2000-2001 and published in 2004, besides alcohol, cannabis and opiates were most commonly used drugs. The prevalence of use among the persons surveyed within the preceding month of the study was : Cannabis 3.0\%, Opiate $0.7 \%$, Any illicit drug 3.6\% and Injecting Drug Users (IDU) $0.1 \%$. Based on above data it was projected that in India, there were approximately 8.7 million cannabis users and 2 million opiate users. It was observed that among current cannabis users $26 \%$ were dependent user and $22 \%$ of current opiate users were dependent users. These figures translate to about 2.3 million cannabisdependent and 0.5 million opiate-dependent individuals. As per the survey and media reports, it was seen that 
the problem is more serious in the border states such as Punjab, Rajasthan, North-Eastern states and mega cities like Delhi, Kolkata, Chennai etc.[4]

\section{Directive Principles of state policy, legal and regulation regimes framework}

The Constitution of India under the Article 47 of Directive Principles of State Policy directs the State to regard the raising of the level of nutrition and the standard of living of its people and the improvement of public health as among its primary duties, and, in particular, to endeavour to bring about prohibition of consumption, except for medicinal purposes, of intoxicating drinks and drugs which are injurious to health. [5]

The Government of India is a signatory to the following three UN Conventions on the subject:-

a) Single Convention on Narcotic Drugs, 1961, as amended by the Protocol of 1972 on Narcotic Drugs.

b) Convention on Psychotropic Substance, 1971, and

c) Convention on Illicit Traffic in Narcotic Drugs and Psychotropic Substance, 1988.

Article 253 of the Constitution empowers the Parliament to legislate for the purpose of discharging obligations under international conventions and foreign treaties. Accordingly, the Narcotics Drugs and Psychotropic Substances Act was enacted in November, 1985, to give effect to the provisions of the existing UN Conventions. It contains stringent provisions for the control and regulation of narcotic drugs and psychotropic substances, and provides an essential framework and appropriate provisions for administrative action. Important provisions of the Act are section 27-Punishment for consumption of small quantity, section 39-Power of court to release certain offenders on probation, section 64A-Immunity from prosecution to addicts volunteering for treatment and section 71-Power of the government to establish centre for identification, treatment etc. besides It provide for prohibition on import / export of preparations containing narcotic drugs /psychotropic substances except authorized by Narcotics Commissioner, no transhipment except with permission of Customs and prohibition on import /export through a post office box.

Drugs and Cosmetic Act 1940 and Rules provide: Rule 97 (1): Labelling of medicines, (c) If it contains a substance specified in Schedule H, and comes within the purview of the [Narcotic Drugs and Psychotropic Substances Act, 1985 (61 of 1985)] be labelled with the symbol NRx which shall be in red and conspicuously displayed on the left top corner of the label, and be also labelled with the following words: Schedule H drug -"Warning: -- To be sold by retail on the prescription of a Registered Medical Practitioner only." Customs Act 1962 provides for regulation for clearance of courier consignments. The Pharmacy Act 1948 provides for Pharmacy Councils and Registration of pharmacists. It would appear that the current legislations are adequate however; regulation of internet pharmacies is needed.

\section{Emerging trend of Pharmaceuticals injecting in India}

Three studies published in the recent past throw some light on use of pharmaceutical compounds by the injecting drug users. Tripathi and Ambekar, 2007, conducted at Jammu, Patiala, Chandigarh (in Northwest) and Imphal and Kohima (in Northeast). The study documented that many places in the country other than the NE part of India have a sizeable number of IDUs. Women and men are differently affected by HIV and understanding gender differences is critical to develop effective response. [6]

Ambekar and Tripathi, 2008, conducted a study "size estimation of IDU in Punjab and Haryana" at multiple sites in India namely Punjab, Haryana and Chandigarh. A total of 3311 male IDUs were surveyed in community settings across 17 districts of Punjab and Haryana. Most subjects were between $18-30$ years of age, and had been injecting drugs for 3 to 7 years. Pharmaceutical preparations injected were buprenorphine, pentazocine and a variety of sedatives (diazepam, promethazine, pheniramine etc.). Most respondents injected daily, multiple times. [7]

Ambekar, Tripathi and Dzuvichu, 2009, in "HIV vulnerability among IDUs their spouses and Children" a total of 300 IDUs were interviewed (150 each in northwest and northeast). The mean age of IDUs was around 34 years, and they had been injecting for about (15 years) in northeast and (11years) in northwest. Most common drugs injected in northwest were a cocktail of various pharmaceutical compounds likeheroin, buprenorphine, propoxyphene etc., while in northeast a large proportion (34\%) reported injecting proxyvon oral capsules. At both the places majority reported injecting daily, multiple times $59 \%$ in northeast and $75 \%$ in northwest. [8]

Table 1 Drug Percentage reporting current (last month) use

\begin{tabular}{|l|l|}
\hline Drugs & Prevalence reporting current (last month) use \\
\hline Other Opioids * & $10.9-12.7 \%$ \\
\hline Sedatives/ Hypnotics** & $5.4-7.2 \%$ \\
\hline Injecting drug User (IDU)*** & $6.2-7.3 \%$ \\
\hline others & $0.7-10.9 \%$ \\
\hline
\end{tabular}


*Drugs included here are buprenorphine, pentazocine, propoxyphene, morphine and pethedine, codeine containing cough syrups and lopiramide.

** Drugs included here are diazepam, alprazolam and other benzodiazepines

*** Others drugs included here are other prescription compounds like ketamine and muscle relaxants.

Abuse of Methylephenidate and other stimulants, barbiturates were not reported.[9]

\section{Commonly Abused Pharmaceuticals injecting \& Related Issues}

India has a very viable pharmaceutical industry, and narcotic drugs and psychotropic substance are produced locally. However, the process of estimating the annual requirements and allotment of quota for psychotropic substances is imprecise, such a situation makes India susceptible to diversion for illegal use. The easy availability of these substances is an advantage to the abusers. The main source of pharmaceuticals of abuse is domestic. Abusers obtain pharmaceuticals mostly from retail pharmacies by misuse of doctors' prescriptions. Pain killers including Opioids (medicines), Codeine containing cough syrups, Sedatives \& hypnotics, Anti-histamines and Ketamine are commonly used in India. The pharmaceuticals drug are often used in combination (cocktails) and as injections, which has several physical harms such as transmission of HIV, Hepatitis C, STIs, Skin infections, Abscess leading physical handicap etc. There is wide regional variation of choice of compound (s) by the users. The sources of these drugs is mainly domestic and from retail pharmacies. Many appear to have shifted from narcotics to pharmaceuticals and there is evidence of poly drug usage. There are regions in India where pharmaceuticals are preferred drugs of choice. [10] There are multiple regulatory agencies which cause overlapping and issues of coordination. The capacity of regulatory and prevention agencies to take up task of bio medical intervention is low. The consumer education and education of pharmacists is abysmally low or virtually nonexistent. There is an urgent need to strengthen capacity of the state drug control authorities and prevention agencies. [11,12]

\section{Issues relating to Injecting Drug Use and HIV prevention for Social Work Practice}

A comprehensive overview of efforts made by the NGOs in member countries who adopted the UN conventions was initiated with UNODC's support (titled 'Beyond 2008') to understand future direction for drug control. It surmised that the focus has largely been on drug supply and enforcement and that a balance was needed between supply and demand reduction approaches. This is true of the Indian situation too, specifically while the NDPS Act's provisions are considered stringent, the low conviction rates and the fact that treatment options are rarely exercised is a cause of concern. [13] Moreover, as persons arrested for use or possession of even small quantities stay in prison for a long time due to procedural delays, it bring into focus issues of human rights, as well as risk of being exposed to or recruited by criminal gangs while in prison especially in the absence of prison based interventions. A study supported by UNODC indicated that $59 \%$ of the convicted prisoners interviewed could have problems related to substance use. [14]

National AIDS control organization (NACO) is the nodal agency for the prevention and control of HIV epidemic in the country and is responsible for the preparation, implementation and monitoring of national strategic plan for HIV/AIDS. The HIV epidemic in the country is concentrated among certain vulnerable population sub-groups like female sex workers (FSWs), men having sex with men (MSMs), injecting drug user (IDUs) and bridge population (truckers/transport workers and migrants). NACO's programme and activities are exclusively directed towards management of IDUs. Interventions for injecting drug users include motivating the subjects to access treatment established in government, NGO and private sectors. Many of them following intervention reduce their drug use and are in need for further treatment and rehabilitation. Additionally, there are certain targeted intervention projects TIs which are supported by NACO and addresses concern of special population subgroups. Interventions for IDUs would also require substitution treatment, referral to detoxification and rehabilitation services. Currently, there are about 268 IDU targeted interventions (TI) sites and NACO supports 75 OST (4810 clients) centres covering more than 177,000 IDUs.

Injecting Drug Use (IDU) has emerged as an important route in the HIV transmission dynamics in India. Through the current number of Injecting drug users (IDUs) in India is 200,000, the HIV prevalence among IDUs is very high as is evident from the recent surveillance exercise by NACO, wherein the HIV prevalence among IDUs was $>9 \%$. Additionally, there are nine districts which have reported $>15 \% \mathrm{HIV}$ prevalence among IDUs in the country. Newer sites where IDU epidemic has been detected also show a high prevalence of HIV among IDUs. These sites are located in states such as Punjab, Haryana, Delhi, Orissa and Bihar. [15]

The increased burden of HIV among IDUs and other blood-borne infections caused by hepatitis $\mathrm{B}(\mathrm{HBV})$ and hepatitis $\mathrm{C}(\mathrm{HCV})$ viruses is due to risk behaviours related to sharing of contaminated needles and syringes as well as through high-risk sexual behaviours such as unprotected sex, unsafe sex under the influence of drugs/alcohol and sex for exchange of drugs.HIV epidemic, initially started by the sharing of contaminated injecting equipment, is spread through sexual transmission from IDUs to others including regular and other 
sexual partners. The interface between drug use and sex work serves as a fuel to the spread of HIV epidemic from the IDU population to sex workers and finally to the general population.

The National AIDS Control Organization (NACO) has adopted the harm reduction (HR) strategy in NACP-III to prevent HIV amongst the IDUs and scaled up interventions through targeted interventions (TI) by non government organizations (NGOs). Evidence supports a comprehensive package of biomedical and behavioural interventions as the optimal HIV prevention strategy for halting HIV among IDUs. The services by the TIs are provided in three tiers: tier 1 services are outreach based interventions and needle syringe exchange programme (NSEP) constituting the backbone of the interventions. Tier 2 is OST based interventions, and tier 3 provides linkages to other important services that are not directly provided under TIs such as Direct Observation Treatment (DOTS centres), Integrated Counselling and Testing Centres(ICTC), Anti Retroviral Treatment (ART centres), reproductive health services and drug use and detoxification and rehabilitation related services.

There is rapid increase in the number of TIs managed by NGOs for IDUs in the country. Data from reports suggests that onetime coverage of about $80 \%$ has been achieved. However, there is no accurate information on the regular coverage and is generally perceived to be very low. Needle and syringe distribution from TI sites have increased substantially. However despite an increase in distribution of needles and syringes, low coverage still remains a challenge. Currently an average of 150 syringes per client/year is being distributed. The average requirement for syringes is estimated to be 300 per client per year. This challenge has to be tackled by the NGO functionaries by developing rapport with clients, understanding the demand of needle and syringes, properly planning the supply through outreach and on site distribution, intensifying the outreach activities by way of understanding vulnerability of clients and proper counselling.

There has been considerable delay in the scale up of Opioid substitution therapy programme. (OST) National AIDS Control Programme III (2007-12) had set out a target of covering $20 \%$ of the IDUs on OST. However, currently, only $2-3 \%$ of the IDUs are on OST. There is a great disparity in the resources provided to NGO OST and Government OST facilities. Clients require a menu of options to choose from medications such as Methadone which is missing. Client retention of OST services is very low (approximately $30 \%$ in 2012 13).Mechanism for tracking OST clients is not fully developed. Currently information is lacking about clients who discontinue OST on their own prior to completion of treatment. Accreditation systems are missing both at public health facilities and NGO settings. This challenge is attributed to the perspective of helping professional towards the issue of drug abuse at public as well as NGO level most of them consider it as a complex problem which is very difficult to be handled. The level knowledge and skills is low amongst the social workers and medical professionals, therefore, it is pertinent to develop learning, sharing and knowledge management systems through capacity building exercises

The other needs of IDUs are not met in the current framework of TIs therefore a system of referral and linkages has been proposed in order to develop provisions significant for HIV prevention such as ART, ICTC, nutrition, homelessness, detoxification and rehabilitation services. The number of IDUs registered with ART centres is too low and adherence to ART medications is also an issue. In addition, several states have performed poorly in overall achievement of HIV testing among IDUs. This has been observed in terms of number of IDU population tested for HIV and gaps between referrals and actual testing at ICTCs. Current data shows that variance in referral-testing gap ranges from $1.3 \%$ to $74 \%$. The linkages with services such nutrition, homelessness, detoxification and rehabilitation services is also non existent. These challenges can be handled by social workers demanding setting up of more detoxification and rehabilitation centres from the government with a provision of free treatment and food, networking with services of schemes which provide food security, initiate kitchen in collaboration with religious organisations and hotels which provide such services in the area. The issue of low ICTC and ART attendance can be handled by enhancing the knowledge, attitude and skills of counsellors and outreach staff of NGOs and ICTC and ART staff.

IDUs among female population have been observed in the North Eastern States and large cities of the country. Therefore capacity building on gender issues and sensitive treatment services are required for the social workers. Role of other substances particularly alcohol in spread of HIV infection is well recognised. Thus, treatment for alcohol dependence and related problems would be important for overall risk reduction and preventing spread of HIV.

Many of these clients are hidden and hard to reach due to stigma and discrimination faced by them. NACO strongly advocates addressing these issues and conduct outreach programme for these communities. At the state level, the efforts initiated by the NACO are decentralised and the services are delivered through State AIDS Control Society (SACs) which are semi autonomous bodies. Therefore SACs would have a major role to play in implementing the national policy and action plan in various states.

At the local level, synergy between IDU-TIs and Integrated Rehabilitation Centres for Addicts Scheme is missing for demand reduction programme of the Ministry of Social Justice and Empowerment. Regular meetings, sharing of examples of best practices, research and programme data can improve coordination. Therefore NGOs can develop strong networking amongst each other. 
There are no comprehensive estimates of Female IDU population in India. About $45 \%$ of the IDUs are either married or cohabiting with a regular sex partners. Extremely low service uptake among regular sex partners of IDUs is reported and high positivity rates are reported among partners in many districts require immediate attention on female IDUs and female partners of IDUs under the programme. It has been observed that condom distribution is only $33 \%$ of the demand. In some states, same -sex activity among IDU has also been observed. Strengthening prevention of transmission of HIV through sexual route among IDU populations is essential. Therefore social workers can promote use of condoms by increasing accessibility, availability and removing barriers to condom use amongst the clients and their partners.

The capacity building efforts have not kept pace with expansion of the IDU TIs programme. This includes capacity gaps across service providers, monitoring and mentoring officers as well as State AIDS Control Societies, technical support units (TSUs) and State Training Resource Centres (STRC) established by NACO. To strengthen capacity, quality and reach of the harm reduction services for IDUs Global fund for fight against AIDS, Tuberculosis and Malaria (GFATM) has funded Indian government and private institution to enhance capacity of staff of NGOs and service delivery institutions like OST centres. The funding under the GFATM supports establishment of strong management and monitoring system and provide for one capacity building officer to each STRC who is primarily responsible to increase capacities of staff of NGOs and other institutions. The programme under implementation has completed more than two years. The findings of mid term evaluation indicates that the programme is moving in the right direction and will achieve goals set by 2015 .

\section{Conclusion}

Social Work professional are employed at all levels from policy making to outreach of clients in India. Over 800 Social Workers are employed in NACP III as programme officers and technical officers with NACO, Capacity building officers with 15 State Training Resource Centres, Technical officers with 11Technical support Units, District managers, supervisors and counsellors with 35 SACs and Project managers, counsellors and outreach staff with 268 NGOs. Employed in a variety of settings, social workers provide individual and family counselling as well as referrals to services in occupational, educational, legal, and health systems.

There are several requisites for working with IDUs, First, there must be a commitment to selfdetermination and treatment modalities that enhance the client's right to make choices, Probing for underlying causes must be abandoned, as it only serves to block change. Assumptions that guide practice in IDUs at micro and mezo level include the following. Deal effectively with the IDUs massive denial system through appropriate confrontation without initially expecting the client to identify and elucidate the problem rationally.

Educate the IDU client and his/her family on the various aspects of the injecting and its impact on his or her quality of life. IDUs know very little, if anything about the bio socio physical effects of injecting. An educational focus initiated by the practitioner helps the client to redefine the problem and move more rapidly toward recovery. Clearly, this calls for an ecological approach that considers salient environmental factors in planning for treatment, discharge, developing occupational skills and minimizing stress and harm. An ecological approach examines factors such as socio cultural influences, age, gender, the presence of social support, the clients' learning style, and orientation to change.

Support networks play an important role in the overall success of intervention programs. Finally, social workers need to consider clients modes of learning and attitudes towards change. They need to design interventions that capitalize on clients strengths. In addition, a number of influences-for example, sub-cultural norms, geographic and financial access to services, staff composition and the cultural relevance of services client believes that treatment alternative is helpful.

At macro levels of intervention social worker should work to strengthen the quality, capacity and reach of the harm reduction services provided through TIs by meticulously planned participatory training and capacity building programmes and technical assistance to NGO staff and providing feedback at policy making levels to improve the services further.

\section{Reference}

[1]. http://socialjustice.nic.in/pdf/NDDRP-march2013.pdf. National drug demand reduction Policy, Ministry of Social Justice and Empowerment, Government of India, 2013

[2]. http://www.searo.who.int/en/Section1 174/Section1 199.htm. Family Health and Research, Mental Health and Substance Abuse.

[3]. http://www.unodc.org/documents/frontpage/CND_Costa_Speech_08_03_10.pdf. International Drug Policy, An unfinished architecture, March 8, 2010

[4]. http://www.unodc.org/india/india_national_survey2004.html. National survey on extent, pattern and trends of drug abuse in India. Ministry of Social Justice and Empowerment and United Nations Office on Drugs and Crime (2004).

[5]. The constitution of India, Government of India,1950.

[6]. BM Tripathi and A Ambekar. A national survey for size estimation of injecting drug users (IDUs) at multiple sites in India, SPYM/DFID, N.Delhi,2007.

[7]. Ambekar, A and BM Tripathi,. Size estimation of injecting drug use in Punjab \& Haryana, SPYM / UNAIDS, N Delhi, 2008

[8]. A Ambekar, A, BM Tripathi and Dzuvichu B. HIV vulnerability among injecting drug users, their spouses and children, SPYM / Plan International, India,2009 
[9]. Drug Abuse Monitoring System, 2007-2012, NDDTC, AIIMS, unpublished report cited in national drug demand reduction policy, Ministry of Social Justice \& Empowerment, GOI, 2013

[10]. http://www.justice.gov/ndic/pubs11/11449/abuse.htm. National Drug Intelligence Center, Pharmaceuticals Drug Threat Assessment November 2004

[11]. http://english.people.com.cn/90001/90782/6436104.html.UNODC study: Pharmaceutical drug abuse increasing in India, June 2008

[12]. http://www.unodc.org/southasia//frontpage/2010/April/abuse-and-trafficking-of pharmaceuticals.html. India-Bangladesh: Trafficking and abuse of pharmaceuticals - an issue of growing public concern, 2010

[13]. UNODC-ROSA. Illicit Use of Prescription Drugs in South Asia, Regional Report, 2010

[14]. Rapid Assessment of substance use among convicted prisoners in Puzhal Prison, T T Ranganathan Clinical Research Foundation (TTRCRF) with support from UNODC, unpublished report, 2009.

[15]. http://nacoonline.org/upload/NACP\%20\%20IV/Consultation\%20I\%20May\%202011/Reports/Strategy\%20document\%20Injecting\%2 0Drug\%20Use final\%20V2.pdf. National AIDS Control Programme phase iv, working group report on injecting drug use, Department of AIDS, Ministry of Health Government of India,2012. 